\title{
Complicación del drenaje endoscópico en un paciente con absceso retroorbitario. Caso clínico
}

\author{
Complication of an endoscopic drainage on a patient \\ with a retro-orbital abscess. Report of a case
}

Paul Boettiger B1', Grettel Martelo P², Jorge Zúñiga P1', Carlos Celedón L¹.

\begin{abstract}
RESUMEN
La rinosinusitis aguda, puede presentar una serie de complicaciones conocidas, un grupo de ellas son las complicaciones orbitarias. Se presenta la historia de un paciente que consultó por proptosis ocular, siendo diagnosticado un absceso subperióstico retroorbitario secundario a una rinosinusitis aguda. Se trató con técnica endoscópica, evoluciona con un hematoma retroorbitario que tuvo que ser drenado con un abordaje mixto, endoscópico y abierto, con buena evolución.

Actualmente las complicaciones orbitarias de la rinosinusitis aguda tienen una baja prevalencia, y así, las complicaciones del drenaje endoscópico son aún menos frecuentes. Se destaca la importancia del conocimiento del manejo tanto endoscópico como abierto.
\end{abstract}

Palabras clave: Hematoma subperióstico retroorbitario, rinosinusitis aguda.

\begin{abstract}
Among acute rhinosinusitis complications, the ones with orbital involvement are relevant. We present a case report of a patient who presented proptosis and was initially diagnosed with a retro ocular abscess as a complication to an acute rhinosinusitis. The abscess was drained with an endoscopic sinus surgery. On the fourth post-surgery day a retro ocular hematoma was diagnosed. The hematoma was treated with a combined open and endoscopic approach with a favorable outcome.

In conclusion, even though orbital complications of rhinosinusitis nowadays have low prevalence, and orbital complications of their endoscopic management are even rarer, it is important for the otorhinolaryngologist to be familiar with their open, endoscopic, or combined surgical treatment.
\end{abstract}

Key words: Retro ocular hematoma, acute rhinosinusitis.

${ }^{1}$ Médico, Servicio de Otorrinolaringología, Hospital Clínico Universidad de Chile.

${ }^{2}$ Médico Cirujano, alumna en estadía de capacitación en Hospital Clínico Universidad de Chile. 


\section{INTRODUCCIÓN}

La rinosinusitis aguda (RSA) constituye una entidad de alta prevalencia a nivel mundial y constituye una de las enfermedades del ámbito otorrinolaringológico más frecuente; la importancia de la RSA no sólo radica en su prevalencia sino en sus complicaciones, graves y potencialmente fatale ${ }^{1-3}$. La incidencia de morbilidad y mortalidad entre los pacientes con complicaciones de RSA oscila entre $5 \%$ y $40 \%{ }^{3,4}$. La rinosinusitis aguda complicada puede desarrollarse como resultado de retraso al iniciar el tratamiento antibiótico oportuno, o por la aparición de resistencia bacteriana, gérmenes más agresivos 0 tratamientos incompletos o inadecuados 5 .

La infección se propaga habitualmente desde los senos etmoidales a través de la lámina papirácea, que separa el complejo etmoidal del contenido orbitario; también puede propagarse a través del piso del seno frontal o del techo del antro maxilar ${ }^{6,7}$. La propagación indirecta es facilitada por las comunicaciones avalvulares entre los plexos venosos orbital y frontal con los senos venosos intracraneales ${ }^{2,6,8}$.

Las complicaciones de la RSA se clasifican en orbitarias e intracraneales. Chandler ${ }^{10}$ clasificó las complicaciones orbitarias en 5 estadios: 1. Celulitis preseptal, 2. Celulitis orbitaria, 3. Absceso subperióstico, 4. Absceso orbitario, y 5. Trombosis del seno cavernos $0^{2,9,10}$. De los anteriormente descritos, el absceso subperióstico y el absceso orbitario, destacan en interés en este caso en particular, porque son los diagnósticos diferenciales a los que nos encontramos enfrentados en el caso que se presenta ${ }^{11,12}$.

Por otra parte, el hematoma retroorbitario es una entidad infrecuente, pero bien descrita. Se observa secundario a traumatismo faciales/orbitarios directos 0 después de una cirugía en esta región ${ }^{8}$. El hematoma espontáneo se observa en pacientes con diátesis hemorrágica o secundaria a congestión venosa orbitaria ${ }^{8,13}$. Rara vez es secundario a sinusitis frontal ${ }^{14}$. Es común en los adolescentes con preponderancia masculina 4,5:1; en relación a las mujeres. El mecanismo del hematoma orbitario es el sangrado continuo de los vasos subgaleales, que tienden a disecar el periostio del hueso. Clínicamente, se presenta con proptosis dolorosa, no pulsátil ${ }^{8}$.

Se presenta el siguiente caso por la baja frecuencia de presentación de las complicaciones orbitarias, asociado a su desenlace, el que debió manejarse con técnica quirúrgica mixta, tanto endoscópica como abierta.

\section{CASO CLINICO}

Paciente masculino de 19 años de edad, sin antecedentes mórbidos de importancia, quien consulta diez días previos a la hospitalización, por un cuadro de fiebre y cefalea. Se plantea el diagnóstico de rinofaringitis y se indica tratamiento sintomático. Evoluciona con aumento de volumen bipalpebral derecho de cinco días de evolución asociado a fiebre, cefalea y un día previo a la consulta se agrega oftalmoparesia, por lo que consulta a oftalmólogo. Se deriva el paciente al Servicio de Urgencia de nuestro hospital, siendo hospitalizado. Se indica tratamiento antibacteriano y se solicita tomografía computarizada (TC) de senos paranasales y órbita con y sin contraste; ese mismo día es evaluado de urgencia por otorrinolaringólogo.

Al examen físico destaca importante proptosis, dolor a la palpación, quemosis, asociado a la incapacidad de realizar supraversión, con visión de colores mantenida. La TC de ingreso al Hospital Clínico de la Universidad de Chile, muestra una imagen sugerente de sinusitis maxiloetmoidal bilateral y absceso subperióstico retroorbitario derecho (Figura 1).

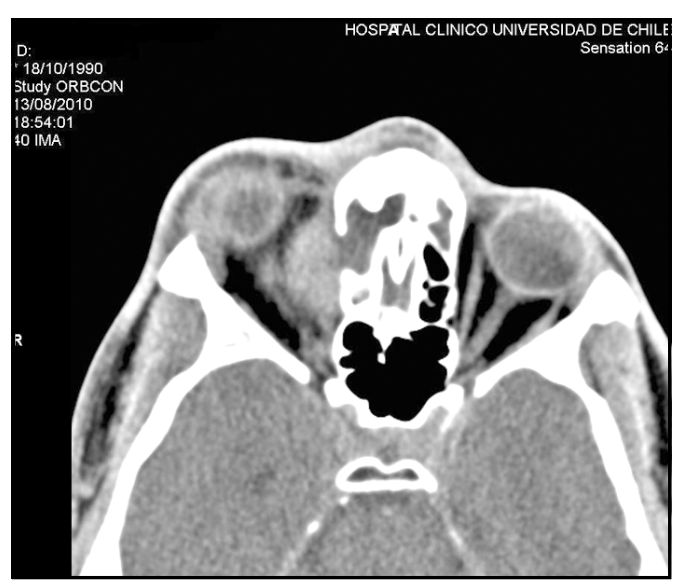

Figura 1. Tomografia computarizada de cavidades paranasales, con contraste, corte axial. Imagen tomada al ingreso del paciente. 
Se realiza drenaje endoscópico de urgencia a las 10 horas de haber ingresado al hospital. En el procedimiento se realiza una antrostomía maxilar derecha, etmoidectomía anterior y posterior derecha, y una antrostomía maxilar izquierda. Finalmente se reseca la lámina papirácea derecha y se realiza drenaje del absceso orbitario; en todos los compartimentos se observa salida de abundante fluido purulento.

El paciente se mantiene hospitalizado con tratamiento endovenoso consistente en ceftriaxona, clindamicina, analgesia y corticoterapia.

Presenta evolución favorable, a las 48 horas se encuentra afebril, con disminución de la proptosis, y a las 72 horas de efectuado el drenaje endoscópico logra movilidad ocular normal.

A las 96 horas de operado se constata una discreta restricción de la supraversión ocular, por lo que se decide solicitar una TC de órbita de control y nueva evaluación por oftalmólogo. En la TC se observa una colección residual supraorbitaria, y en el control oftalmológico se confirma la limitación de la movilidad en supraversión (Figura 2). Se sugiere biopsia y estudio inmunológico.

Veinticuatro horas después, se constata una manifiesta restricción de la supraversión, con importante dolor a la palpación del párpado superior, asociado a aumento de volumen y tensión en la órbita. Se decide realizar drenaje de urgencia com-

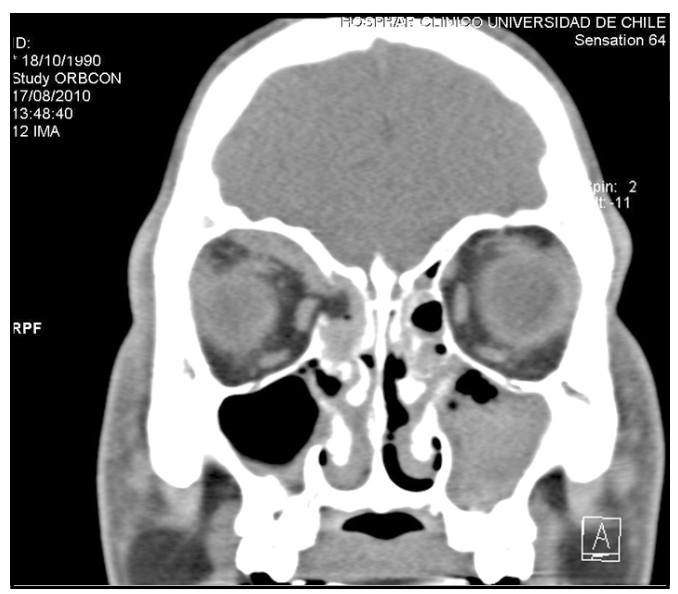

Figura 2. Tomografia computarizada de cavidades paranasales, con contraste, corte coronal, imagen tomada al tercer dia posdrenaje endoscópico. binado, endoscópico y abierto, con diagnóstico presuntivo de absceso orbitario recidivado.

Se realiza un abordaje endoscópico, incluyendo una septoplastía a objeto de ampliar el campo operatorio. En etmoides se observa protrusión de la grasa orbitaria y del músculo recto medial. Al no poder acceder por esta vía a la órbita, se decide un abordaje externo. Se realiza una incisión supraciliar, introduciéndose a la órbita. Se encuentra un hematoma retrobulbar, protrusión de la mucosa y músculo hacia el etmoides. El músculo se reposiciona. Se realiza hemostasia identificándose una pequeña arteria supraorbitaria con sangrado activo. Se contiene con Surgicel Fibrillar ${ }^{\circledR}$. Se deja un drenaje tipo Penrose en la cavidad orbitaria sin fijación, a través de la fosa nasal, por vía endoscópica.

Se indica alta al cuarto día de la segunda intervención. El paciente evoluciona satisfactoriamente, afebril, y con movilidad ocular normal, se indica de completar tratamiento antibacteriano oral por 21 días.

En controles posteriores, se constata evolución favorable, con visión y movilidad ocular normal. El informe anatomopatológico revela actividad alérgica e infección aguda concomitante. El estudio inmunológico fue normal.

\section{DISCUSIÓN}

El caso presentado, corresponde a un paciente con cuadro de absceso subperióstico secundario a una rinosinusitis aguda drenado de urgencia, por vía endoscópica, quien presentó en su evolución, una complicación posiblemente secundaria a la primera cirugía, caracterizada por sangrado y posterior formación de un hematoma retroorbitario, originado en una pequeña arteria supraorbital.

Fue difícil identificar la real causa del sangrado, considerando que la arteria sangrante se encontraba lejos del primer acceso endoscópico y era inaccesible a través de éste ${ }^{5}$. Además existen reportes de sangrado espontáneo asociado a una rinosinusitis crónica que provocaron un hematoma retroorbitario ${ }^{8}$.

El hematoma presentó sus primeros signos de existencia a las 72 horas, manifestándose clínicamente por una leve limitación en la 
supraversión ocular, planteando el diagnóstico diferencial entre una recidiva del absceso y la formación de un hematoma.

La clínica asociada fue escasa: dolor exquisito a la palpación del párpado superior, y ausencia de fiebre, pudiendo esta última estar enmascarada por el uso de analgesia endovenosa. Analizados en retrospectiva, estos signos podrían habernos sugerido el diagnóstico diferencial, por que las imágenes tomográficas, en el caso del paciente fueron indistinguibles, entre colección purulenta y hematoma ${ }^{14}$.

El tratamiento de elección de esta complicación en la literatura concuerda con lo realizado en nuestro caso. El acceso abierto supraorbital asociado a la cirugía endoscópica parece ser la mejor alternativa ${ }^{7-9}$

\section{CONCLUSIÓN}

Hoy en día la formación de los especialistas se ha enfocado en la cirugía endoscópica. Este caso clínico refuerza la práctica del abordaje externo que, aisladamente 0 combinado al acceso endoscópico, puede ser útil en el tratamiento de las complicaciones.

Se nos plantea el desafío del reconocimiento precoz de complicaciones de la cirugía endoscópica.

\section{BIBLIOGRAFÍA}

1. Rosenfeld R, Andes D, Bhattacharyya $\mathrm{N}$ et al. Clinical Practice Guideline: Adult Sinusitis. Otol Head and Neck Surgery. 2007; 137: S1-S31.

2. Masood A, Moumoulidis I, Panesar J. Acute Rhinosinusitis in Adults: an Update on Current Management. Postgrad Med J 2007; 83: 402-8.

3. Ali A, Kurien $M$, Mathews $S$, Mathew J. Complications of Acute Infective Rhinosinusitis:
Experience from a Developing Country. Singapore Med J 2005; 46(10): 540-4.

4. Howe L, Jones N. Guidelines for the Management of Periorbital Cellulitis/Abscess. Clin Otolaryngol 2004; 29: 725-8.

5. Clarke W. Periorbital and Orbital Cellulitis in Children. Paediatr Child Health 2004; 9(7): 471-2.

6. Reid J. Complications of Pediatric Paranasal Sinusitis. Pediatr Radiol 2004; 34: 933-42.

7. Rahbar R, Robson C, Petersen R et al. Management of Orbital Subperiosteal Abscess in Children. Arch Otolaryngol Head Neck Surg 2001; 127: 281-6.

8. Woo KI, Kim YD. Subperiosteal hematoma of the orbit associated with sinusitis. Korean $\mathrm{J}$ Ophthalmol 1997; 11(2): 118-22.

9. Tanna N, Preciado D, Clary M, Choi S. Surgical Treatment of Subperiosteal Orbital Abscess. Arch Otolaryngol Head Neck Surg 2008; 134(7): 764-7.

10. Chandler JR, Landenbrunner DJ \& Stevens ER. The pathogenesis of orbital complications in acute sinusitis. Laryngoscope 1970; 80: 141428.

11. Martin-Hirsch D, Habashi S, Hinton A, Kotecha B. Orbital Cellulitis. Archives of Emergency Medicine 1992; 9: 143-8.

12. Davis J, Steams M. Orbital Complications of Sinusitis: Avoid Delays in Diagnosis. Postgrad Med J 1994; 70: 108-10.

13. Velasco A, Cassiano R, Pereira F, Dos Santos A, Anselmo-Lima W, Da Silva R. Orbital Complications of Acute Rhinosinusitis: A New Classification. Rev Bras Otorrinolaringol 2007; 73(5): 95.

14. Eutis H, Mafee M, Walton C, Mondonca J. MR Imaging and CT of Orbital Infections and Complications in Acute Rhinosinusitis. Radiol Clin North Am 1998; 36(6): 1165-83. 